Trauma Berufskrankh 2009 • 11:127 DOI 10.1007/s10039-009-1548-4

Online publiziert: 14. September 2009

๑) Springer Medizin Verlag 2009

\author{
A.Wentzensen \\ Berufsgenossenschaftliche Unfallklinik Ludwigshafen
}

\title{
Rechnergestütztes Operieren
}

Moderne Schnittbildverfahren haben vor 30 Jahren eine neue Ära in der Diagnostik für die Chirurgie eingeleitet. Die Einführung des rechnergestützten Operierens vor 15 Jahren ermöglichte es dann, die diagnostische Präzision in die operative Handlung zu übertragen.

Die Ziele sind klar: Die Präzision chirurgischer Eingriffe muss bei Indikationen, bei denen Defizite bestehen, verbessert, die Sicherheit von Interventionen, etwa bei minimal invasiven Verfahren, gesteigert werden. Ein gutes Beispiel ist die $\mathrm{Na}$ vigation in Verbindung mit intraoperativer 3-D-Bildgebung an der Wirbelsäule. Hier sind erste Ergebnisse ermutigend. Besonderes Plus: Bei wesentlich verbesserter Präzision kann die Strahlenbelastung für Patient und OP-Personal reduziert werden.

Viele Kliniken in Deutschland, Europa und den USA wenden Navigationssysteme an. Hauptsächlich werden sie bei der Implantation von Kunstgelenken im Bereich der Hüfte und vor allem am Kniegelenk eingesetzt. Endoprothetik ist gut planbar, in den Kliniken können Teams geschult werden. Das ermöglicht den Einsatz in klinischer Routine.

Anders beim frischen Trauma: Hier ist die Planungszeit begrenzt, sind geschulte Teams nur eingeschränkt verfügbar. Auch gibt es Defizite in der navigierten Kontrolle der Reposition bei der operativen Frakturversorgung. Erfahrungen zeigen, dass die Navigation beim Trauma nicht einfach so in den OP eingeführt werden kann. Vorbereitung und Planung im OP müssen umgestellt, teilweise auch neue Instrumente entwickelt werden. Von klinischer Routine ist man hier noch weit entfernt.

\section{() Rechnergestütztes Operieren führt eine virtuelle Welt in das chirurgische Umfeld ein}

Bereiche, in denen das rechnergestützte Operieren chirurgische Techniken verbessert, müssen noch benannt werden. So macht es zum Beispiel keinen Sinn, die Navigation für einfache Vorgänge wie das Einbringen einer distalen Verriegelung zu verwenden. Nur als Komplettlösung, die Reposition und Implantatplatzierung einschließt, kann sie Vorteile bringen. Das ist etwa in topographisch schwierigen Regionen der Fall wie der operativen Versorgung von Beckenverletzungen und der Resektion von Tumoren im Beckenbereich.

Die Schlüsselrolle für einen erfolgreichen Einsatz der Navigation spielt die Schulung der Anwender. Fehlschläge beim navigierten Operieren sind meist auf Anwendungsfehler und Fehlinterpretation des Chirurgen zurückzuführen. Bewährt haben sich Teams, die in den Kliniken die Navigation anwenden, kontinuierlich weiterentwickeln und auch die Verantwortung dafür übernehmen. So lassen sich technische Probleme mindern und es lässt sich die Effizienz erhöhen.

Rechnergestütztes Operieren führt gleichsam eine virtuelle Welt in das chirurgische Umfeld ein. Es verbessert die Übersicht beim Operieren, macht aber aus Anfängern nicht automatisch Experten. Der Schlüssel für gute Ergebnisse ist immer der erfahrene Chirurg. Die Systeme müssen noch einfacher und anwenderfreundlicher werden. Sie sollten stabil und zuverlässig funktionieren und Softwareingenieure im OP überflüssig machen. Und sie sollten wirtschaftlich trag- bar sein, gerade unter den heutigen ökonomischen Rahmenbedingungen. Rechnergestütztes Operieren muss für den $\mathrm{Pa}$ tienten einen Nutzen bringen und gleichzeitig die Anschaffungskosten sowie den zeitlichen und personellen Mehraufwand rechtfertigen.

Im vorliegenden $\mathrm{Heft}$ werden typische Anwendungsbeispiele und neue Entwicklungen mit klinischer Relevanz demonstriert.

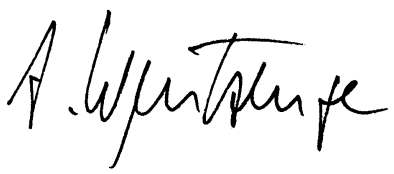

Prof. Dr. A.Wentzensen

\section{Korrespondenzadresse}

Prof. Dr. A.Wentzensen

Berufsgenossenschaftliche Unfallklinik, Ludwig-Guttmann-Str. 13,

67071 Ludwigshafen

Andreas.Wentzensen@urz.uni-heidelberg.de 